SISSA $17 / 2007 / \mathrm{EP}$

\title{
Particle decay in Ising field theory with magnetic field 1
}

\author{
Gesualdo Delfino \\ International School for Advanced Studies (SISSA), \\ via Beirut 2-4, 34014 Trieste, Italy \\ Istituto Nazionale di Fisica Nucleare, sezione di Trieste, Italy
}

\begin{abstract}
The scaling limit of the two-dimensional Ising model in the plane of temperature and magnetic field defines a field theory which provides the simplest illustration of non-trivial phenomena such as spontaneous symmetry breaking and confinement. Here we discuss how Ising field theory also gives the simplest model for particle decay. The decay widths computed in this theory provide the obvious test ground for the numerical methods designed to study unstable particles in quantum field theories discretized on a lattice.
\end{abstract}

\footnotetext{
${ }^{1}$ To appear in the proceedings of the XVth International Congress on Mathematical Physics, Rio de Janeiro, 6-11 August 2006.
} 


\section{Ising field theory}

Quantum field theory provides the natural tool for the characterization of universality classes of critical behavior in statistical mechanics. While the general ideas based on the renormalization group apply to any dimension (see e.g. [1]), the two-dimensional case acquired in the last decades a very special status. Indeed, after the exact description of critical points was made possible by the solution of conformal field theories [2, it appeared that also specific directions in the scaling region of two-dimensional statistical systems can be described exactly [3]. Additional insight then comes from perturbation theory around these integrable directions [4].

The two-dimensional Ising model plays a basic role in the theory of critical phenomena since when Onsager computed its free energy and provided the first exact description of a second order phase transition [5]. Its scaling limit in the plane of temperature and magnetic field defines a field theory - the Ising field theory - which provides the simplest example of non-trivial phenomena such as spontaneous symmetry breaking and confinement [6]. Here we will discuss how Ising field theory also yields the simplest model for particle decay [7].

The Ising model is defined on a lattice by the reduced Hamiltonian

$$
E=-\frac{1}{T} \sum_{\langle i, j\rangle} \sigma_{i} \sigma_{j}-H \sum_{i} \sigma_{i}, \quad \sigma_{i}= \pm 1
$$

so that the partition function is $Z=\sum_{\left\{\sigma_{i}\right\}} e^{-E}$. On a regular lattice in more than one dimension, the model undergoes, for a critical value $T_{c}$ of the temperature and for vanishing magnetic field $H$, a second order phase transition associated to the spontaneous breakdown of spin reversal symmetry.

In two dimensions the scaling limit of (1) is described by the Ising field theory with action

$$
\mathcal{A}=\mathcal{A}_{C F T}-\tau \int d^{2} x \varepsilon(x)-h \int d^{2} x \sigma(x) .
$$

Here $\mathcal{A}_{C F T}$ is the action of the simplest reflection-positive conformal field theory in two dimensions, which corresponds to the Ising critical point [2]. The spin operator $\sigma(x)$ with scaling dimension $X_{\sigma}=1 / 8$ and the energy operator $\varepsilon(x)$ with scaling dimension $X_{\varepsilon}=1$ are, together with the identity, the only relevant operators present in this conformal theory. The couplings $h$ and $\tau$ account for the magnetic field and the deviation from critical temperature, respectively.

The field theory (2) describes a family of renormalization group trajectories flowing out of the critical point located at $h=\tau=0$ (Fig. 1). Since the coupling conjugated to an operator $\Phi$ has the dimension of a mass to the power $2-X_{\Phi}$, the combination

$$
\eta=\frac{\tau}{|h|^{8 / 15}}
$$

is dimensionless and can be used to label the trajectories. In particular, the low- and hightemperature phases at $h=0$ and the critical isotherm $\tau=0$ correspond to $\eta=-\infty,+\infty, 0$, respectively. 


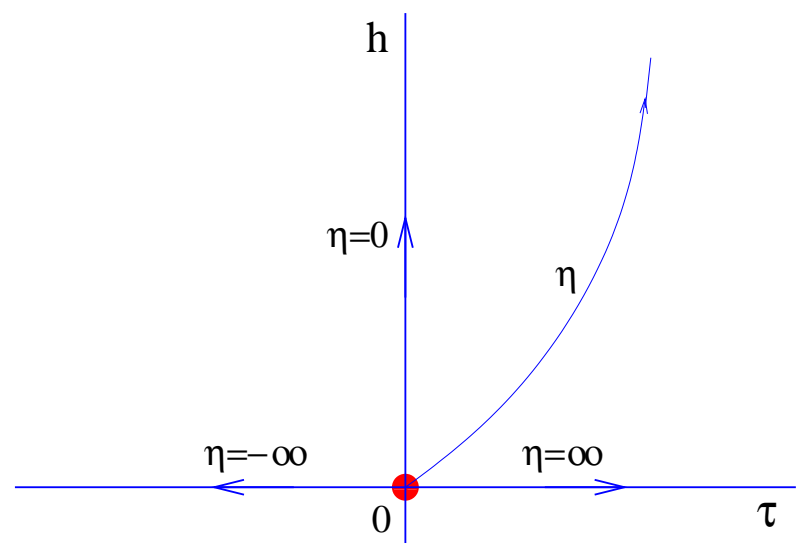

Figure 1: The Ising field theory (2) describes a one-parameter family of renormalization group trajectories (labelled by $\eta$ ) flowing out of the critical point located at $\tau=h=0$.

\section{Evolution of the mass spectrum}

Under analytic continuation to imaginary time the euclidean field theory (2) defines a 1+1dimensional relativistic theory allowing for a particle interpretation.

It is well known that the action (2) with $h=0$ describes a free neutral fermion with mass $m \sim|\tau|$. While in the disordered phase $\tau>0$ this fermionic particle corresponds to ordinary spin excitations, in the spontaneously broken phase $\tau<0$ it describes the kinks interpolating between the two degenerate ground states of the system (Fig. 2a). In the euclidean interpretation the space-time trajectories of the kinks correspond to the domain walls separating regions with opposite magnetization.

A small magnetic field switched on at $\tau<0$ breaks explicitely the spin reversal symmetry and removes the degeneracy of the two ground states (Fig. 2b). To first order in $h$ the energy density difference between the two vacua is

$$
\Delta \mathcal{E} \simeq 2 h\langle\sigma\rangle,
$$

where $\langle\sigma\rangle$ is the spontaneous magnetization at $\tau=0$. With the symmetry broken the kinks are

no longer stable excitations. An antikink-kink pair, which was a two-particle asymptotic state of the theory at $h=0$, now encloses a region where the system sits on the false vacuum (Fig. $2 \mathrm{~b}$ ). The need to minimize this region induces an attractive potential

$$
V(R) \simeq \Delta \mathcal{E} R
$$

( $R$ is the distance between the walls) which confines the kinks and leaves in the spectrum of the theory only a string of antikink-kink bound states $A_{n}, n=1,2, \ldots$, whose masses

$$
m_{n}=2 m+\frac{(\Delta \mathcal{E})^{2 / 3} z_{n}}{m^{1 / 3}}, \quad h \rightarrow 0
$$

are obtained from the Schrodinger equation with the potential (5). The $z_{n}$ in (6) are positive numbers determined by the zeros of the Airy function, $\operatorname{Ai}\left(-z_{n}\right)=0$. This non-relativistic 


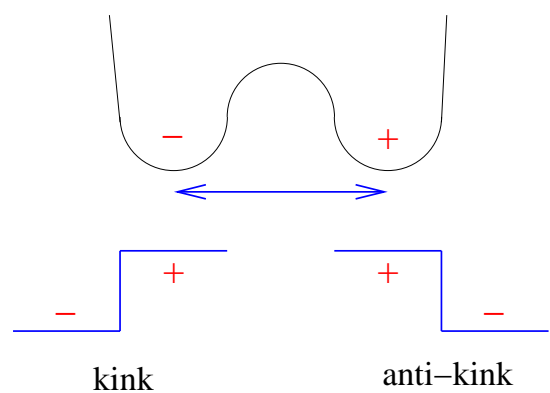

(a)

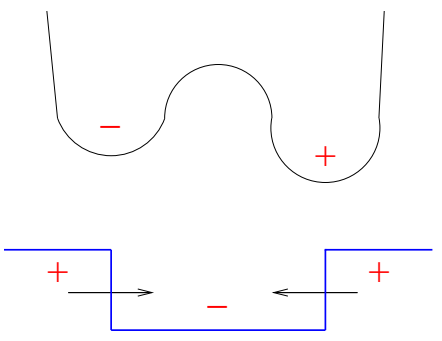

(b)

Figure 2: The free energy and the kink excitations in the spontaneously broken phase (a). A small magnetic field removes the degeneracy of the ground states and confines the kinks (b).

approximation is exact in the limit $h \rightarrow 0$ in which $m_{n}-2 m \rightarrow 0$. The spectrum (6) was first obtained in [6] from the study of the analytic structure in momentum space of the spin-spin correlation function for small magnetic field. Relativistic corrections to (6) have been obtained more recently 1 in $[8]$.

The particles $A_{n}$ with mass larger than twice the lightest mass $m_{1}$ are unstable. It was conjectured in [6] that the number of stable particles descreases as $\eta$ increases from $-\infty$, until only $A_{1}$ is left in the spectrum of asymptotic particles as $\eta \rightarrow+\infty$. The particle $A_{1}$ would then be the free fermion of the theory at $\eta=+\infty$. According to this scenario, for any $n>1$ there should exist a value $\eta_{n}$ for which $m_{n}$ crosses the decay threshold $2 m_{1}$, so that the particle $A_{n}$ becomes unstable for $\eta>\eta_{n}$. The natural expectation is that the values $\eta_{n}$ decrease as $n$ increases, in such a way that the $\tau$ - $h$ plane is divided into sectors with a different number of stable particles as qualitatively shown in Fig. 3. The trajectories corresponding to the values $\eta_{n}$ are expected to densely fill the plane in the limit $\eta \rightarrow-\infty$.

This pattern has been confirmed by numerical investigations of the spectrum of the field theory (2) for all values of $\eta$ [4, 8, 9]. For $\eta \rightarrow-\infty$ the particles $A_{n}$ with $n$ large can be studied within the semiclassical approximation and their decay widths have been obtained in [10, 9$]$.

\section{Particle decay off the critical isotherm}

The critical isotherm $\eta=\tau=0$ must lie within the sector in which the theory has, generically, three stable particles (Fig. 3). To understand this we must recall that A. Zamolodchikov showed in [3] that the theory (2) with $\tau=0$ is integrable and computed its exact $S$-matrix. He found that the spectrum along this integrable trajectory consists of eight stable particles $A_{1}, \ldots, A_{8}$

\footnotetext{
${ }^{1}$ See also [9] which appeared after this talk was given.
} 


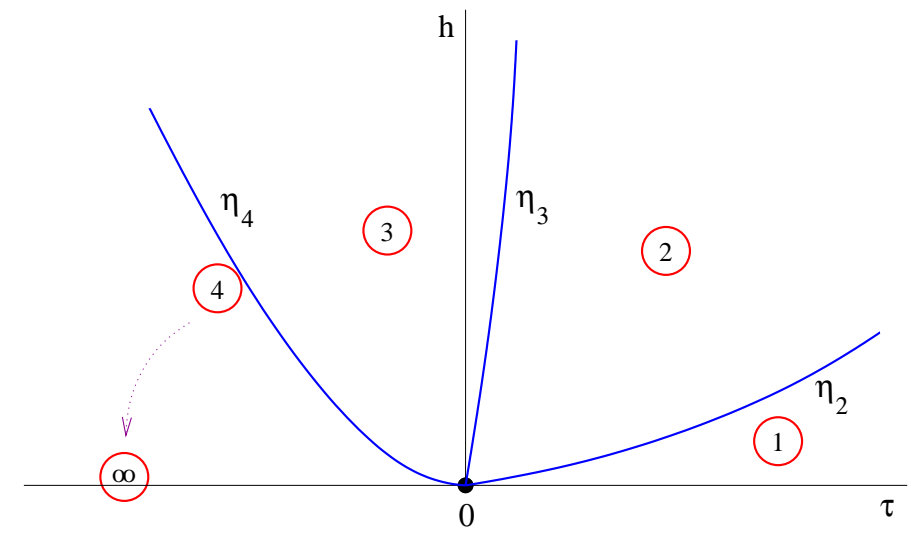

Figure 3: Expected evolution of the mass spectrum as a function of $\eta$. In the sector in between $\eta_{n}$ and $\eta_{n+1}$ the theory possesses $n$ stable particles (numbers in the circles).

with masses

$$
\begin{aligned}
& m_{1} \sim h^{8 / 15} \\
& m_{2}=2 m_{1} \cos \frac{\pi}{5}=(1.6180339887 . .) m_{1} \\
& m_{3}=2 m_{1} \cos \frac{\pi}{30}=(1.9890437907 . .) m_{1} \\
& m_{4}=2 m_{2} \cos \frac{7 \pi}{30}=(2.4048671724 . .) m_{1} \\
& m_{5}=2 m_{2} \cos \frac{2 \pi}{15}=(2.9562952015 . .) m_{1} \\
& m_{6}=2 m_{2} \cos \frac{\pi}{30}=(3.2183404585 . .) m_{1} \\
& m_{7}=4 m_{2} \cos \frac{\pi}{5} \cos \frac{7 \pi}{30}=(3.8911568233 . .) m_{1} \\
& m_{8}=4 m_{2} \cos \frac{\pi}{5} \cos \frac{2 \pi}{15}=(4.7833861168 . .) m_{1} .
\end{aligned}
$$

A peculiarity of this spectrum is that only the lightest three particles lie below the lowest decay threshold $2 m_{1}$. The remaining five have the phase space to decay and certainly are not prevented to do so by internal symmetries (the magnetic field leaves no internal symmetry in the Ising model). It is easy to see that, while there is nothing wrong with the stability of the particles above threshold along this integrable trajectory, they must necessarily decay as soon as a deviation, however small, from the critical temperature breaks integrability [7]. Figure 4 shows the bound state poles and the unitarity cuts of the elastic scattering amplitudes $S_{11}$ and $S_{12}$ in the complex plane of the relativistic invariant $s$ (square of the center of mass energy). We know from [3] that at $\tau=0$ the scattering channel $A_{1} A_{1}$ produces the first three particles as bound states (Fig. 4a), while the channel $A_{1} A_{2}$ produces the first four (Fig. 4b). The absence of inelastic scattering in integrable theories allows only for the unitarity cut associated to the elastic processes. When integrability is broken (i.e. as soon as we move away from $\tau=0$ ), however, the inelastic channels and the associated unitarity cuts open up. In particular, the process $A_{1} A_{2} \rightarrow A_{1} A_{1}$ acquires a non-zero amplitude, so that the threshold located at $s=4 m_{1}^{2}$ 
becomes the lowest one also in the $A_{1} A_{2}$ scattering channel (Fig. 4c). Since the pole associated to $A_{4}$ is located above this threshold, it can no longer remain on the real axis, which in that region is now occupied by the new cut. The position of the pole must then develop an imaginary part which, according to the general requirements for unstable particles [11, is negative and brings the pole through the cut onto the unphysical region of the Riemann surface. The other particles above threshold, which appear as bound states in other amplitudes at $\tau=0$, decay through a similar mechanism. We see then that, beacuse of integrability, the trajectory $\eta=0$ corresponds to an isolated case with eight stable particles inside a range of values of $\eta$ in which only the particles $A_{1}, A_{2}, A_{3}$ are stable.

These decay processes associated to integrability breaking can be studied analytically through the form factor perturbation theory around integrable models [4. Indeed, if the action of the perturbed integrable theory is

$$
\mathcal{A}_{\text {integrable }}+\lambda \int d^{2} x \Psi(x)
$$

the perturbative series in $\lambda$ can be expressed in terms of the matrix elements of the perturbing operator $\Psi$ on the asymptotic particle states (Fig. 5). These matrix elements can be computed exactly in the unperturbed, integrable theory exploiting analyticity constraints [12, 13] supplemented by operator-dependent asymptotic conditions at high energies [14, 15, 16].

For our present purposes we then look at the action (2) as the integrable trajectory $\tau=0$ perturbed by the energy operator $\varepsilon(x)$ and must compute corrections in $\tau$. The matrix elements of an operator $\Phi(x)$ in the unperturbed theory can all be related to the form factor 2

$$
F_{a_{1} \ldots a_{n}}^{\Phi}\left(\theta_{1}, \ldots, \theta_{n}\right)=\left\langle 0|\Phi(0)| A_{a_{1}}\left(\theta_{1}\right) \ldots A_{a_{n}}\left(\theta_{n}\right)\right\rangle
$$

where $|0\rangle$ is the vacuum state and the asymptotic states are built in terms of the eight particles which are stable at $\tau=0$.

To lowest order in $\tau$ the corrections to the real and imaginary parts of the masses come from the perturbative terms in Fig. 6 and are given by [4]

$$
\begin{gathered}
\delta \operatorname{Re} m_{c}^{2} \simeq-2 \tau f_{c}, \\
\operatorname{Im} m_{c}^{2} \simeq-\tau^{2} \sum_{a \leq b, m_{a}+m_{b} \leq m_{c}} 2^{1-\delta_{a b}} \frac{\left|f_{c a b}\right|^{2}}{m_{c} m_{a}\left|\sinh \theta_{a}^{(c a b)}\right|}, \quad c=4,5
\end{gathered}
$$

with

$$
\begin{gathered}
f_{c}=F_{c c}^{\varepsilon}(i \pi, 0), \\
f_{c a b}=F_{c a b}^{\varepsilon}\left(i \pi, \theta_{a}^{(c a b)}, \theta_{b}^{(c a b)}\right) ;
\end{gathered}
$$

$\theta_{a}^{(c a b)}$ is determined by energy-momentum conservation at the vertices in Fig. $6 \mathrm{~b}$.

\footnotetext{
${ }^{2}$ The energy and momentum of the particles are parameterized in terms of rapidities as $\left(p^{0}, p^{1}\right)=$ $\left(m_{a} \cosh \theta, m_{a} \sinh \theta\right)$.
} 


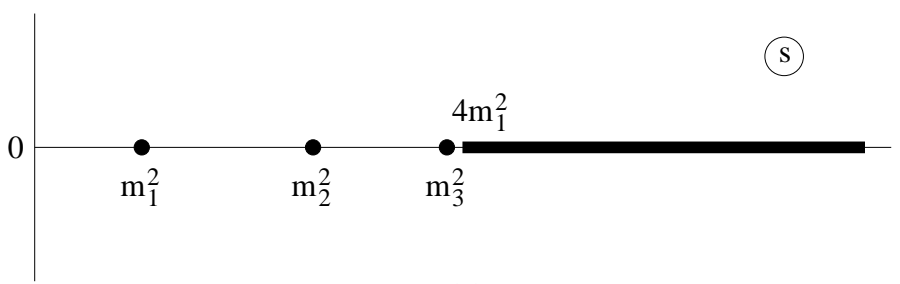

(a)

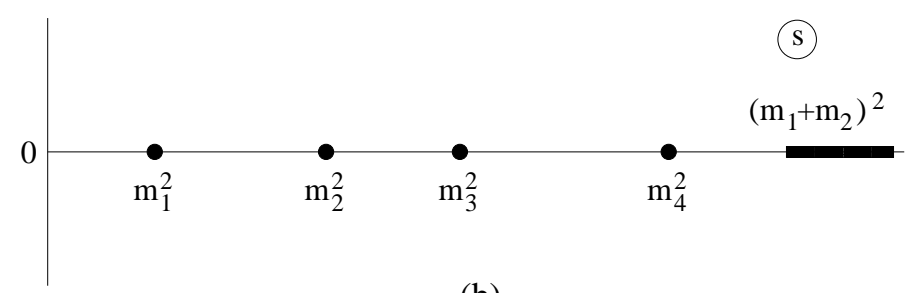

(b)

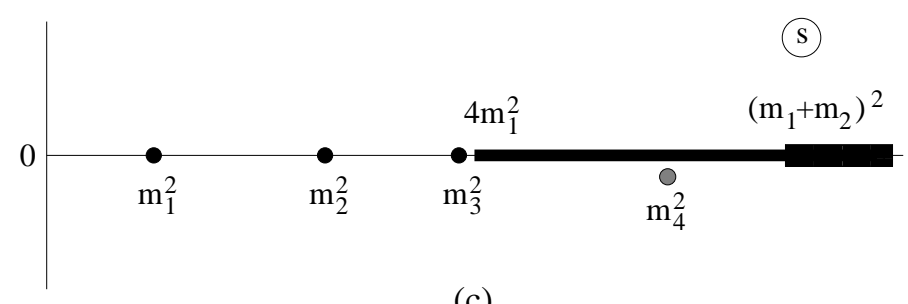

(c)

Figure 4: Poles and unitarity cuts for the elastic scattering amplitudes $S_{11}$ and $S_{12}$ in the integrable case $\tau=0$, (a) and (b), respectively, and for $\tau$ slightly different from zero (c). In (c) the particle $A_{4}$ became unstable and the associated pole moved through the cut into the unphysical region.

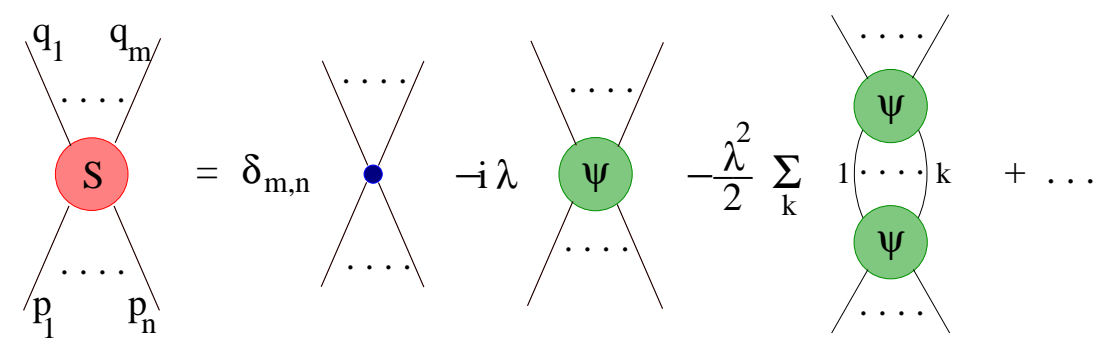

Figure 5: Perturbative expansion for a scattering amplitude in the theory (8)). The matrix elements of the perturbing operator $\Psi$ can be computed exactly in the unperturbed, integrable theory. 


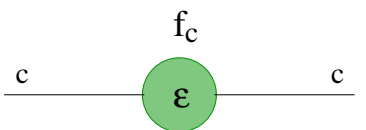

(a)

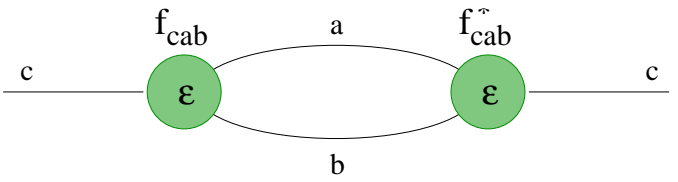

(b)

Figure 6: Terms determining the leading corrections to the real (a) and imaginary (b) parts of the masses in Ising field theory at small $\tau$. For $c>5$ also diagrams with more than two particles in the intermediate state contribute to the imaginary part.

\begin{tabular}{|r|r|r|}
\hline & \multicolumn{1}{|c|}{$\hat{\sigma}$} & \multicolumn{1}{c|}{$\hat{\varepsilon}$} \\
\hline \hline$F_{1}$ & $-0.640902 .$. & $-3.706584 .$. \\
$F_{2}$ & $0.338674 .$. & $3.422288 .$. \\
$F_{3}$ & $-0.186628 .$. & $-2.384334 .$. \\
$F_{4}$ & $0.142771 .$. & $2.268406 .$. \\
$F_{5}$ & $0.060326 .$. & $1.213383 .$. \\
$F_{6}$ & $-0.043389 .$. & $-0.961764 .$. \\
$F_{7}$ & $0.016425 .$. & $0.452303 .$. \\
$F_{8}$ & $-0.003036 .$. & $-0.105848 .$. \\
\hline
\end{tabular}

Table 1: One-particle form factors for the operators $\sigma$ and $\varepsilon$ in Ising field theory at $\tau=0$ [14, 17]. The rescaling implied by the notation $\hat{\Phi} \equiv \Phi /\langle\Phi\rangle$ ensures that the results in the table are universal.

The available decay channels for the particles above threshold are determined by the spectrum (7):

$$
\begin{aligned}
& A_{4} \rightarrow A_{1} A_{1} \\
& A_{5} \rightarrow A_{1} A_{1}, A_{1} A_{2} \\
& A_{6} \rightarrow A_{1} A_{1}, A_{1} A_{2}, A_{1} A_{3}, A_{1} A_{1} A_{1},
\end{aligned}
$$

and similarly for $A_{7}$ and $A_{8}$. For $c>5$ the sum in (11) must be completed including the contributions of the decay channels with more than two particles in the final state.

One- and two-particle form factors for Ising field theory at $\tau=0$ have been computed in [14, 17] (see [18] for a review). Table 1 contains the complete list of one-particle matrix elements for the relevant operators. The results for the lightest particle are compared in Table 2 with the result of numerical diagonalization of the transfer matrix on the lattice [19]. Three-particle form factors have been computed in [7] in order to determine the imaginary parts (11). 


\begin{tabular}{|c||c|c|}
\hline & Field theory & Lattice \\
\hline$F_{1}^{\hat{\sigma}}$ & $-0.640902 .$. & $-0.6408(3)$ \\
$F_{1}^{\hat{\hat{\varepsilon}}}$ & $-3.70658 .$. & $-3.707(7)$ \\
\hline
\end{tabular}

Table 2: Lightest-particle form factors at $\tau=0$ from integrable quantum field theory [14, 17] and from numerical diagonalization of the transfer matrix [19].

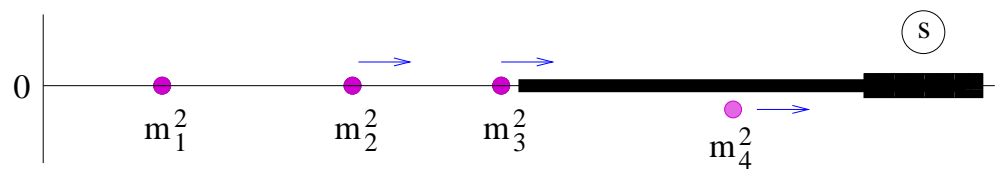

Figure 7: Evolution of the mass spectrum as predicted by (16) as $\tau$ increases from $0 . A_{2}$ and $A_{3}$ approach the threshold and will decay for positive values of $\tau$; $A_{4}$ has already become unstable for $\tau<0$ and moves further away from the threshold.

The available results relevant for the leading mass corrections are

$$
\begin{aligned}
& f_{1}=(-17.8933 . .)\langle\varepsilon\rangle \quad\left|f_{411}\right|=(36.73044 . .)|\langle\varepsilon\rangle| \\
& f_{2}=(-24.9467 . .)\langle\varepsilon\rangle \quad\left|f_{511}\right|=(19.16275 . .)|\langle\varepsilon\rangle| \\
& f_{3}=(-53.6799 . .)\langle\varepsilon\rangle \quad\left|f_{512}\right|=(11.2183 . .)|\langle\varepsilon\rangle| \\
& f_{4}=(-49.3206 . .)\langle\varepsilon\rangle
\end{aligned}
$$

where $\langle\varepsilon\rangle$ is taken at $\tau=0$. The ratios

$$
\lim _{\eta \rightarrow 0} \frac{\delta \operatorname{Re} m_{a}^{2}}{\delta \mathcal{E}}=2 \frac{f_{a}}{\langle\varepsilon\rangle}
$$

where $\delta \mathcal{E}$ is the variation of the vacuum energy density, are completely universal and particularly easy to check numerically. These predictions for the variations of the real part of the masses of the first four particles have been confirmed numerically both in the continuum [4, 9] and on the lattice [20]. Using the result3 21$]$

$$
\langle\varepsilon\rangle=-(2.00314 . .)|h|^{8 / 15},
$$

it easy to check that the the sign of the variations

$$
\delta r_{a}=-\frac{\tau f_{1}}{m_{1} m_{a}}\left(r_{a}^{2}-\frac{f_{a}}{f_{1}}\right)+O\left(\tau^{2}\right)
$$

of the mass ratios $r_{a}=\operatorname{Re} m_{a} / m_{1}$ agrees with the McCoy-Wu scenario (Fig. 7).

The above values of $f_{a b c}$ give [7]

$$
\operatorname{Im} m_{4}^{2} \simeq(-173.747 . .) \tau^{2},
$$

\footnotetext{
${ }^{3}$ The value (15) refers to the normalization of $\varepsilon$ in which $\langle\varepsilon(x) \varepsilon(0)\rangle \rightarrow|x|^{-2}$ as $|x| \rightarrow 0$.
} 


$$
\operatorname{Im} m_{5}^{2} \simeq(-49.8217 . .) \tau^{2},
$$

and in turn the decay widths and lifetimes

$$
\Gamma_{a}=-\frac{\operatorname{Im} m_{a}^{2}}{m_{a}}, \quad t_{a}=\frac{1}{\Gamma_{a}}
$$

of the particles $A_{4}$ and $A_{5}$. The lifetime ratio

$$
\lim _{\tau \rightarrow 0} \frac{t_{4}}{t_{5}}=0.23326 .
$$

is universal, as well as the branching ratio for $A_{5}$, which decays at $47 \%$ into $A_{1} A_{1}$ and for the remaining fraction into $A_{1} A_{2}$.

It appears from (20) that $A_{5}$ lives more than four times longer than $A_{4}$, somehow in contrast with the expectation inherited from accelerator physics that, in absence of symmetry obstructions, heavier particles decay more rapidly. Notice, however, that in $d$ dimensions the width for the decay $A_{c} \rightarrow A_{a} A_{b}$ is

$$
\Gamma_{c \rightarrow a b} \propto g^{2}\left|f_{a b c}\right|^{2} \Phi_{d},
$$

where $g$ is the perturbative parameter, $f_{a b c}$ the form factor and

$$
\Phi_{d} \sim \int \frac{d^{d-1} \vec{p}_{a}}{p_{a}^{0}} \frac{d^{d-1} \vec{p}_{b}}{p_{b}^{0}} \delta^{d}\left(p_{a}+p_{b}-p_{c}\right) \sim \frac{|\vec{p}|^{d-3}}{m_{c}}
$$

the phase space $\left(\vec{p}=\vec{p}_{a}=-\vec{p}_{b}\right)$. For fixed decay products, $|\vec{p}|$ increases with $m_{c}$ and in $d=2$ suppresses the phase space, in contrast with what happens in $d=4$. The results for the vertices $f_{a b c}$ indicate that in our case the dynamics further enhances the increase of $t_{c}$ with $m_{c}$.

\section{Unstable particles in finite volume}

The issue of obtaining numerical checks of theoretical predictions for decay processes is made particularly interesting by the difficulty of characterizing unstable particles in the finite volume [22]. The problem is particularly relevant for lattice gauge theories.

In two dimensions energy spectra can be obtained by numerical diagonalization of a truncated Hamiltonian on a cylinder geometry. The signature of particle decay on the cylinder is clear. At an integrable point, when the energy levels are plotted as a function of the circumference $R$ of the cylinder, the line corresponding to a particle above threshold crosses infinitely many levels which belong to the continuum when $R=\infty$ (Fig. 8). Once integrability is broken, this line "disappears" through a removal of level crossings and a reshaping of the lines associated to stable excitations (Fig. 9).

One way of extracting the decay width from such a spectrum is the following [22]. Consider energy levels corresponding to states with two particles of mass $m$ and momenta $p$ and $-p$, sufficiently close to the threshold $E=2 m$ that the particles can only scatter elastically, with scattering amplitude $S(p)=\exp i \delta(p)$. On the circle, $p$ is quantized by the condition $e^{i R p} S(p)=$ 1 , or equivalently

$$
R p+\delta(p)=2 \pi n
$$




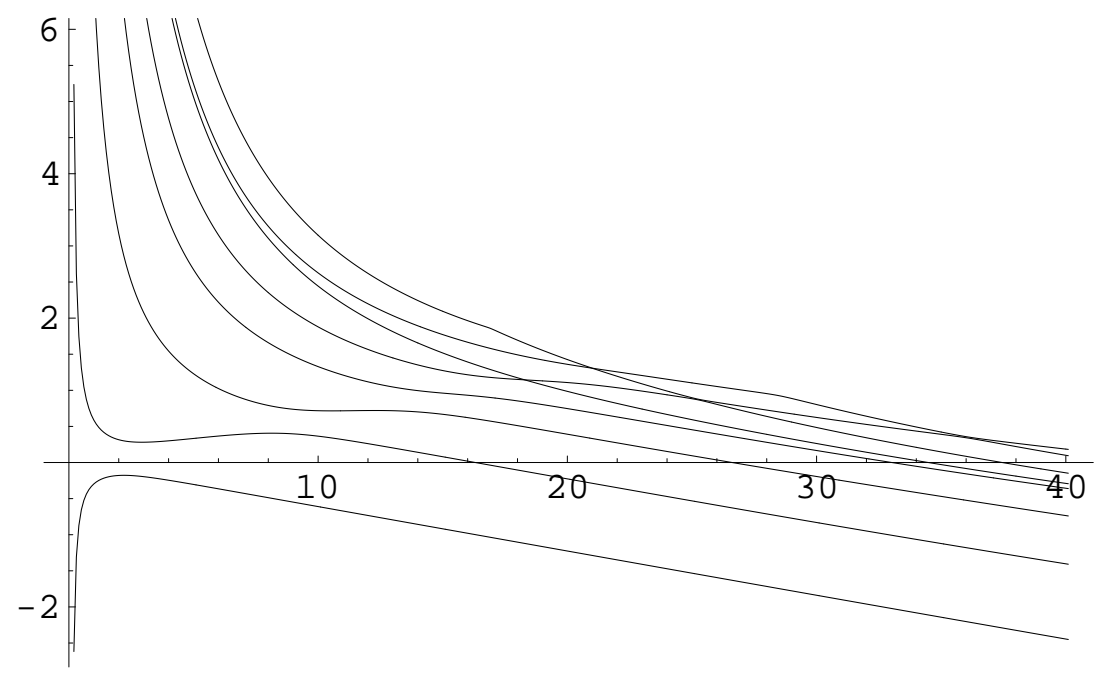

Figure 8: First eight energy levels of the finite volume Hamiltonian of Ising field theory at $\tau=0$ as functions of $r=m_{1} R$ (from Ref. [4]). At $r=40$, starting from the bottom, the levels are identified as the ground state, the first three particle states $A_{1}, A_{2}$ and $A_{3}$, three scattering states $A_{1} A_{1}$, the particle above threshold $A_{4}$. Crossings between the line associated to the latter and the scattering states are visible around $r=18, r=25$ and $r=36$.

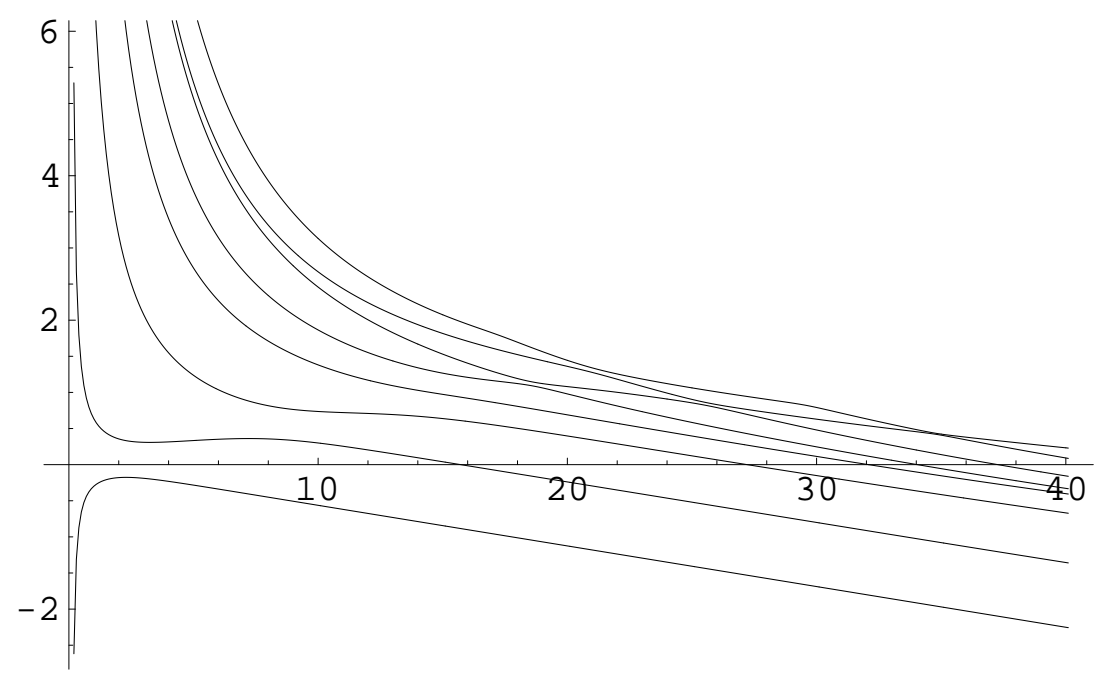

Figure 9: First eight energy levels of the finite volume Hamiltonian of Ising field theory slightly away from $\tau=0$ (from Ref. [4]). Observe the splitting of the crossings pointed out in the previous figure. 


\begin{tabular}{|c|c|c|}
\hline$\left|\hat{f}_{\text {cab }}\right|$ & Exact & Numerical \\
\hline$\left|\hat{f}_{411}\right|$ & $36.730 .$. & $36.5(3)$ \\
$\left|\hat{f}_{511}\right|$ & $19.163 .$. & $19.5(9)$ \\
\hline
\end{tabular}

Table 3: The values of the three-particle vertices $\hat{f}_{a b c} \equiv f_{a b c} /\langle\varepsilon\rangle$ obtained numerically in [23] compared with the exact predictions of [7].

with $n$ labelling the states. Hence $\delta(p)$ can be determined from the measure of

$$
E(R)=2 \sqrt{p^{2}+m^{2}} .
$$

A narrow resonance of mass $m_{c}$ and width $\Gamma$ can then be fitted through the Breit-Wigner formula

$$
\delta(p)=\delta_{0}(p)+\delta_{B W}(p), \quad \delta_{B W}=-i \ln \frac{E-m_{c}-i \Gamma / 2}{E-m_{c}+i \Gamma / 2},
$$

$\delta_{0}$ being a smooth background.

An alternative method was proposed in [7]. At an integrable point consider a particle with mass $m_{c}>m_{a}+m_{b}$. The states

$$
\left|A_{c}(p=0)\right\rangle \equiv|1\rangle, \quad\left|A_{a}(p) A_{b}(-p)\right\rangle \equiv|2\rangle
$$

are degenerate on the cylinder at a crossing point $R^{*}$. When integrability is broken by a perturbation

$$
V=\lambda \int_{0}^{R} d x \Psi(x)
$$

the energy splitting at $R^{*}$ is, to lowest order in perturbaton theory,

$$
\Delta E=\sqrt{\left(V_{11}-V_{22}\right)^{2}+4\left|V_{12}\right|^{2}},
$$

where $V_{i j}=\langle i|V| j\rangle$. Choosing a crossing point at $R^{*}$ large enough, the $V_{i j}$ 's are well approximated by the infinite volume matrix elements. In this way the decay vertices $V_{12}$ can be obtained from measures of the energy splittings.

Both methods have been used in [23] to measure the decay widths of the first two particles above threshold in Ising field theory close to the critical temperature from numerical diagonalization of a truncated Hamiltonian, directly in the continuum limit [24]. The results are shown in Table 3 together with the predictions of form factor perturbation theory discussed in the previous section. It seems obvious that any numerical method designed to measure decay widths in lattice models should first of all be able to recover these predictions for the two-dimensional Ising model.

Acknowledgments. I thank P. Grinza and G. Mussardo, my co-authors of Ref. [7] on which this talk is mainly based. This work is partially supported by the ESF grant INSTANS and by the MIUR project "Quantum field theory and statistical mechanics in low dimensions". 


\section{References}

[1] J.L. Cardy, Scaling and renormalization in statistical physics, Cambridge University Press, 1996.

[2] A.A. Belavin, A.M. Polyakov and A.B. Zamolodchikov, Nucl. Phys. B 241 (1984) 333.

[3] A.B. Zamolodchikov, Advanced Studies in Pure Mathematics 19 (1989) 641; Int. J. Mod. Phys. A 3 (1988) 743.

[4] G. Delfino, G. Mussardo and P. Simonetti, Nucl. Phys. B 473 (1996) 469.

[5] L. Onsager, Phys. Rev. 65 (1944) 117.

[6] B.M. McCoy and T.T. Wu, Phys. Rev. D 18 (1978) 1259.

[7] G. Delfino, P. Grinza and G. Mussardo, Nucl. Phys. B 737 (2006) 291.

[8] A.B. Zamolodchikov and P. Fonseca, J. Stat. Phys. 110 (2003) 527.

[9] A.B. Zamolodchikov and P. Fonseca, hep-th/0612304.

[10] S.B. Rutkevich, Phys. Rev. Lett. 95 (2005) 250601.

[11] R.J. Eden, P.V. Landshoff, D.I. Olive and J.C. Polkinghorne, The analytic S-matrix, Cambridge University Press, 1966.

[12] M. Karowski, P. Weisz, Nucl. Phys. B 139 (1978) 455.

[13] F.A. Smirnov, Form Factors in Completely Integrable Models of Quantum Field Theory, World Scientific, 1992.

[14] G. Delfino and G. Mussardo, Nucl. Phys. B 455 (1995) 724.

[15] G. Delfino, P. Simonetti and J.L. Cardy, Phys. Lett. B 387 (1996) 327.

[16] G. Delfino and G. Niccoli, Nucl. Phys. B 707 (2005) 381; J. Stat. Mech. (2006) P03008.

[17] G. Delfino and P. Simonetti, Phys. Lett. B 383 (1996) 450.

[18] G. Delfino, J. Phys. A 37 (2004) R45.

[19] M. Caselle and M. Hasenbusch, Nucl. Phys. B 579 (2000) 667.

[20] P. Grinza and A. Rago, Nucl. Phys. B 651 (2003) 387.

[21] V.A. Fateev, S. Lukyanov, A.B. Zamolodchikov and Al.B. Zamolodchikov, Nucl. Phys. B 516 (1998) 652.

[22] M. Luscher, in "Fields, strings and critical phenomena", Proc. Les Houches Summer School, ed. E. Brezin and J. Zinn-Justin, North Holland, 1989. 
[23] B. Pozsgay and G. Takacs, hep-th/0604022.

[24] V.P. Yurov and Al.B. Zamolodchikov, Int. J. Mod. Phys. A6 (1991) 4557. 